\title{
One-dimensional Molecular Crystal of Phthalocyanine Confined into Single-Walled Carbon Nanotubes
}

\author{
Laurent Alvarez, ${ }^{1, *}$, Fallou Fall ${ }^{2}$, Anouar Belhboub ${ }^{1,3}$, Rozenn Le Parc ${ }^{l}$, Yann Almadori ${ }^{1,2}$, \\ Raul Arenal ${ }^{4,5}$, Raymond Aznar ${ }^{l}$, Philippe Dieudonné-George ${ }^{1}$, Patrick Hermet ${ }^{6}$, Abdelali Rahmani ${ }^{3}$, Bruno \\ Jousselme $^{7}$, Stéphane Campidelli ${ }^{7}$, Julien Cambedouzou $^{8}$, Takeshi Saito ${ }^{9}$ and Jean-Louis Bantignies ${ }^{1}$
}

(1) Laboratoire Charles Coulomb (L2C), UMR 5221 CNRS-Université de Montpellier, Montpellier, F-France.

Laurent.Alvarez@univ-montp2.fr, 33467143541

(2) Physikalisches Institut, Albert-Ludwigs-Universitat, 79104 Freiburg, Germany

(3) Laboratoire d'Etude des Matériaux Avancés et Applications (LEM2A), Faculté des Sciences, Université Moulay Ismaïl, Boîte Postale 11201, Zitoune, 50000 Meknes, Morocco

(4) Laboratorio de microscopias avanzadas (LMA), Instituto de Nanociencia de Aragon (INA), U. Zaragoza, c/ Mariano Esquillor s/n, 50018 Zaragoza, Spain

(5) Fundacion ARAID,50018 Zaragoza, Spain

(6) Institut Charles Gerhardt Montpellier, UMR 5253 CNRS-UM2-ENSCM-UM1, Université Montpellier 2, Place E. Bataillon, 34095 Montpellier, France

(7) CEA-Saclay, IRAMIS, NIMBE, Laboratoire d'Innovation en Chimie des Surfaces et Nanosciences (LICSEN), 91191 Gif-sur-

Yvette Cedex, France

(8) Institut de Chimie Séparative de Marcoule, UMR 5257 CEA/CNRS/ENSCM/Université Montpellier 2, 30207 Bagnols sur Cèze, France

(9) Nanotube Research Center, National Institute of Advanced Industrial Science and Technology (AIST), Tsukuba 305-8565, Japan

Keywords: Encapsulation, Chromophores, x-ray Diffraction, Raman, supramolecular organization

ABSTRACT: New 1D hybrid nano-systems are elaborated with metallated or metal free phthalocyanine molecules encapsulated into the hollow core of single-wall carbon nanotubes. The x-ray diffraction experiments coupled to simulation allow evidencing the $1 \mathrm{D}$ structural organization of the molecules inside the nanotubes. The angle between the molecule ring and the nanotubes axis is close to $32^{\circ}$ as determined from our DFT calculations. Confined molecules display Raman spectra hardly altered with respect to the bulk phase, suggesting a rather weak interaction with the tubes. For comparison, non-covalent functionalization at the outer surface of the tube is also investigated. The vibrational properties of the molecules functionalized at the outer surface of tubes display important modifications. A significant curvature of the phthalocyanine is induced by the interaction with the tube walls, leading to change of the central atom position within the molecular ring, in good agreement with our first principle calculations. 


\section{INTRODUCTION}

Phthalocyanine $(\mathrm{Pc})$ are $18 \pi$-electron aromatic molecules which can accommodate a wide range of atoms in their central cavity to form metallocomplexes (MPc). ${ }^{1}$ Initially used as dyes and pigments, MPc molecules have attracted an increasing interest as molecular building blocks for catalytic and opto-electronics. ${ }^{2-5}$ In addition, depending on the nature of the central atom, MPc can carry a magnetic moment so that the molecules can be thought as nanomagnets, opening new routes for spintronics ${ }^{6}$ and data storage devices at the molecular level. ${ }^{7}$ It has been demonstrated that the optical or magnetic properties can be tuned not only by changing the central atom but also by altering their structural properties, ${ }^{1}$ providing practical routes to fit different requirements. More precisely, the molecular orientation plays a key role in charge transport, light absorption, energy level alignment at interfaces in organic semiconductors ${ }^{8}$ and magnetic properties. ${ }^{1}$ For instance, edge-to-edge molecular organization favors in-plane charge transport whereas face-to-face arrangement favors out-of-plane charge transport. ${ }^{8}$

Thus, controlling the structural properties is a key point for potential applications. In this context, carbon nanotubes can be used to constrain phthalocyanine molecule configuration and create original 1D assemblies affording new and tunable magnetic and opto-electronic properties. New hybrid systems containing phthalocyanine derivatives and carbon nanotubes or graphene have been developed during the last years. ${ }^{6,9-21}$ Nevertheless, no endohedral functionalization of single-walled carbon nanotubes (NTs) with MPc has been reported. There are only few studies dealing with the insertion of MPc inside large multi-walled nanotubes with an average inner diameter around $25 \AA^{20,21}$ No internal order was evidenced.

Here, we report on the encapsulation of MPc (free-base $\left(\mathrm{H}_{2}\right)$ and metallated $\left(\mathrm{Zn}^{\mathrm{II}}\right.$ and $\left.\mathrm{Fe}^{\mathrm{II}}\right)$ ) into single-walled carbon nanotubes. These nanotubes display several advantages such as metallic or seminconducting properties and diameters $(\sim 14.5 \AA)$ hardly larger than the molecule size. Thus, the molecules are expected to perfectly fit in the hollow core of NTs, allowing important confinement effects. We clearly evidence a 1D structure, showing that carbon NTs strongly influence the long range structural properties of MPc. The physical interactions in the hybrid system are discussed and we show that they are dominated by molecule-molecule interaction. For comparison, functionalization at the outer surface of the tube is also explored. In this case, molecule-nanotube interactions are dominant, affecting the local structure of the phthalocyanine. Two different single-walled carbon nanotubes are studied, exhibiting average diameters of $14.5 \AA$ (NT14) and $17 \AA$ (NT17).

\section{EXPERIMENTAL METHODS:}

Iron, Zinc and metal free phthalocyanine molecules (respectively $\mathrm{FePc}, \mathrm{ZnPc}$ and $\mathrm{H}_{2} \mathrm{Pc}$ ) are purchased from Sigma Aldrich ( $a r$ for as received). They are purified $(p f)$ by a temperature treatment at $500^{\circ} \mathrm{C}$ under dynamic high vacuum. The purification step consists in heating the molecules at their sublimation temperature. The objective is twofold: i) purification of the sample by separating the molecules from impurities and ii) the molecule after the purification step has undergone the same temperature treatment as for the encapsulation protocol (see below) and it is thus similar to the encapsulated ones. Consequently, it can be used as reference 
with respect to the confined one. The different samples are named $\mathrm{MPc}_{\mathrm{ar}}$ and $\mathrm{MPc}_{\mathrm{pf}}(\mathrm{M}=\mathrm{Fe}, \mathrm{Zn}$ or $\mathrm{H}_{2}$ ). Single walled carbon nanotubes are either purchased from Carbon Solution Company or supplied by National Institute of Advanced Industrial Science and Technology (AIST), Japan. They are synthesized by the enhanced direct injection pyrolytic synthesis (eDIPS) method. ${ }^{22}$ The nanotubes of the two different sources display average diameter of $1.4 \pm 0.2 \mathrm{~nm}$ and $1.7 \pm 0.2 \mathrm{~nm}$ respectively. Therefore, the samples will be named respectively NT14 and NT17 in the following. As received (ar) closed nanotubes are used for the outer surface functionalization ( $\pi$-stacking). Purified and annealed (pf) open nanotubes are used for molecule encapsulation into the hollow core of nanotubes. Thus, the different samples are named NT14 ar, $\mathrm{NT}_{1} 4_{\mathrm{pf}}, \mathrm{NT} 17_{\mathrm{ar}}$ and NT1 $17_{\mathrm{pf}}$.

Synthesis of the hybrid system: A mixture of nanotubes and MPc within ratio 1:2 was outgased under $6 \times 10^{-6}$ mbar at ambient temperature for $2 \mathrm{~h}$ followed by a thermal treatment at $200{ }^{\circ} \mathrm{C}$. The glass-tube was then sealed and heated homogeneously in a furnace at $500^{\circ} \mathrm{C}$ during 24 hours. After this treatment, excess of phthalocyanine was removed by outgasing at $500^{\circ} \mathrm{C}$ at $6 \times 10^{-6}$ mbar. Samples were then washed five times with N-Methyl-2-Pyrrolidone (NMP). As received nanotubes give rise to non-covalent functionalization at the outer surface of the nanotubes $\left(\mathrm{MPc} / \mathrm{NT}_{\mathrm{ar}}\right)$ whereas purified nanotubes lead to the encapsulation process (MPc@NT $\mathrm{pf}_{\mathrm{p}}$. NT can be either NT14 or NT17.

Transmission electron microscopy and spatial-resolved electron energy loss spectroscopy: The transmission electron microscopy (TEM) samples were prepared by dispersing the NTs powders in ethanol. The dispersions were ultrasonicated and subsequently deposited on holey carbon $3 \mathrm{~mm}$ copper grids.

High resolution TEM was performed employing an imaging-side aberration-corrected FEI Titan-Cube microscope working at $80 \mathrm{keV}$, equipped with a Cs corrector (CETCOR from CEOS GmbH).

Spatial-resolved electron energy loss spectroscopy (EELS) measurements were performed on probe-corrected scanning TEM (STEM) FEI Titan Low-Base 60-300 operating at 80keV (fitted with a X-FEG ${ }^{\circledR}$ gun and Cs-probe corrector (CESCOR from CEOS GmbH)). Furthermore, in order to avoid the effects of electron beam damage, these measurements have been performed using a liquid-nitrogen-cooled cryo-holder at $-170^{\circ} \mathrm{C}$. EEL spectra were recorded using the spectrum-imaging (SPIM in $2 \mathrm{D}$ or spectrum-line (SPLI) in $1 \mathrm{D}) \operatorname{mode}^{23,24}$ in a Gatan GIF Tridiem ESR 865 spectrometer. The convergent semi-angle was of $25 \mathrm{mrad}$, the collection semi-angle was of $35 \mathrm{mrad}$ and the energy resolution $\sim 1.2 \mathrm{eV}$.

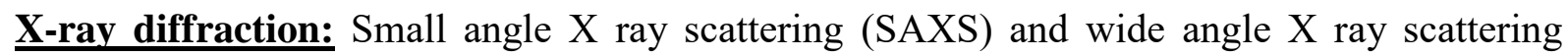
(WAXS) experiments were performed with an in-house setup of the Laboratoire Charles Coulomb, "Réseau X et gamma", Université Montpellier, France. A high brightness low power $\mathrm{X}$ - ray tube, coupled with aspheric multilayer optic (GeniX ${ }^{3 \mathrm{D}}$ from Xenocs) was employed. It delivers an ultralow divergent beam $(0.5 \mathrm{mrad})$. Scatterless slits were used to give a clean 0.8 $\mathrm{mm}$ beam diameter (35 Mphotons/s) at the sample. We worked in a transmission configuration and scattered intensity was measured by a Schneider 2D imageplate detector prototype, at a 
distance of $1,9 \mathrm{~m}$ from the sample for SAXS configuration, $0.2 \mathrm{~m}$ from the sample for WAXS configuration. All Intensities were corrected by transmission and the empty cell contribution was substracted.

The calculated diffraction pattern features bundles of 16 NTs with mean diameter of $1.7 \mathrm{~nm}$. A diameter distribution of FWHM $0.17 \mathrm{~nm}$ was used. The nanotubes are filled with balls of diameter $0.7 \mathrm{~nm}$ and containing 290 electrons, mimicking Fe-phtalocyanines rotating around the long axis of carbon nanotubes. The balls were spaced of a distance compatible with the position observed for the diffraction peak that emerges in the pattern of FePc@NT17 pr. We chose to consider the inserted complexes as balls of smaller diameter than the complex for 2 reasons. First, the electron distribution is not homogeneous in the FePc complex, and is concentrated near the complex center. Second, we assume a rotation around the SWNT axis, which is the only direction possible considering the steric hindrance imposed by the surrounding nanotube. For such a rotation, and considering the symmetry of the electron distribution in the FePc complex, the mean electron distribution can be represented by a ball containing all the electrons of complex, i.e. 290 electrons.

Raman spectroscopy: Micro-Raman experiments have been performed in a back scattering geometry, using the fixed 633, and $660 \mathrm{~nm}$ excitation wavelengths. In order to prevent the heating of the tubes and oligomers, the laser power was adjusted at $100 \mu \mathrm{W}$ with a spot diameter of about $3 \mu \mathrm{m}$ using a 50X objective. The resolution is about $2 \mathrm{~cm}^{-1}$. At least four spatially separated area of each sample were probed to ensure homogeneity. The precision in the peak position measurements is $\pm 1 \mathrm{~cm}^{-1}$.

Computational details : The calculations were performed in the framework of the density functional theory (DFT) as implemented in the SIESTA code. ${ }^{25}$ Two models have been studied: (i) an encapsulation model where we considered the guest molecule inside a $(22,0)$ nanotube of $12.78 \AA$ length and a diameter of $17.22 \AA$, and (ii) a $\pi$-stacked model where the guest molecule is $\pi$-stacked on a $(17,0)$ or $(22,0)$ nanotube of $29.82 \AA$ length and a diameter of 13.31 and 17.22 $\AA$, respectively. The periodic conditions were applied along the tube axis and the vacuum size was of $20 \AA$ in the two other directions to avoid interactions between adjacent tubes.

Here, the guest molecules are metal-free phthalocyanine $\left(\mathrm{H}_{2} \mathrm{Pc}\right)$ and zinc phthalocyanine $(\mathrm{ZnPc})$. The atomic positions were relaxed at fixed lattice parameters using a conjugate gradient until the maximum residual force on the atoms was smaller than $0.02 \mathrm{eV} / \AA$. Exchangecorrelation effects were handled within the generalized gradient approximation (GGA) as proposed by Perdew, Burke and Ernzerhof. ${ }^{26}$ Core electrons are replaced by nonlocal normconserving pseudopotentials. ${ }^{27} \mathrm{~A}$ double zeta singly polarized basis set (DZP) of localized atomic orbitals was used for the valence electrons. ${ }^{28}$ Their cutoff radii were determined from an energy shift of $50 \mathrm{meV}$ by localization. A mesh cutoff of 300 Ry was used for the reciprocalspace expansion of the total charge density and the Brillouin zone was sampled using three $\mathrm{k}$ points along the nanotube axis. Van der Waals corrections between the nanotubes and the guest molecules were considered according to the Grimme approach. ${ }^{29}$ This approach (DFT-D) consists in adding a semi-empirical dispersion potential (a simple pair-wise force field) to the conventional Kohn-Sham DFT energy. 


\section{RESULTS AND DISCUSSION:}

Transmission electron microscopy: Figures 1.a corresponds to a high resolution (HRTEM) micrograph of single-walled nanotubes after the encapsulation step. All the nanotube seem filled with a very good efficiency. However, it is worth mentioning that from this kind of HRTEM micrographs it is not possible to deduce the nature of the confined material, neither to obtain any chemical information. Thus, in order to go further in the analysis of these materials and to know where the MPc molecules are localized, we have developed SR-EELS. This technique is a very powerful tool to investigate such nanomaterials at (sub-) nanometer scale. 30-34 Structural and chemical properties of bundle of single-walled nanotubes have also been investigated. We have acquired a spectrum-image (SPIM) in the whole area showed in the high angle annular dark field (HAADF) image (Figure 1.b).

Figure 1.c displays two EEL spectra extracted in the blue and red areas marked on Figure 1.b, respectively. Each of these two EEL spectra corresponds to the sum of 9 spectra $(3 \times 3$ probe positions of the SPIM). Carbon K edge at $\sim 285 \mathrm{eV}$ is present in both of these two EEL spectra.
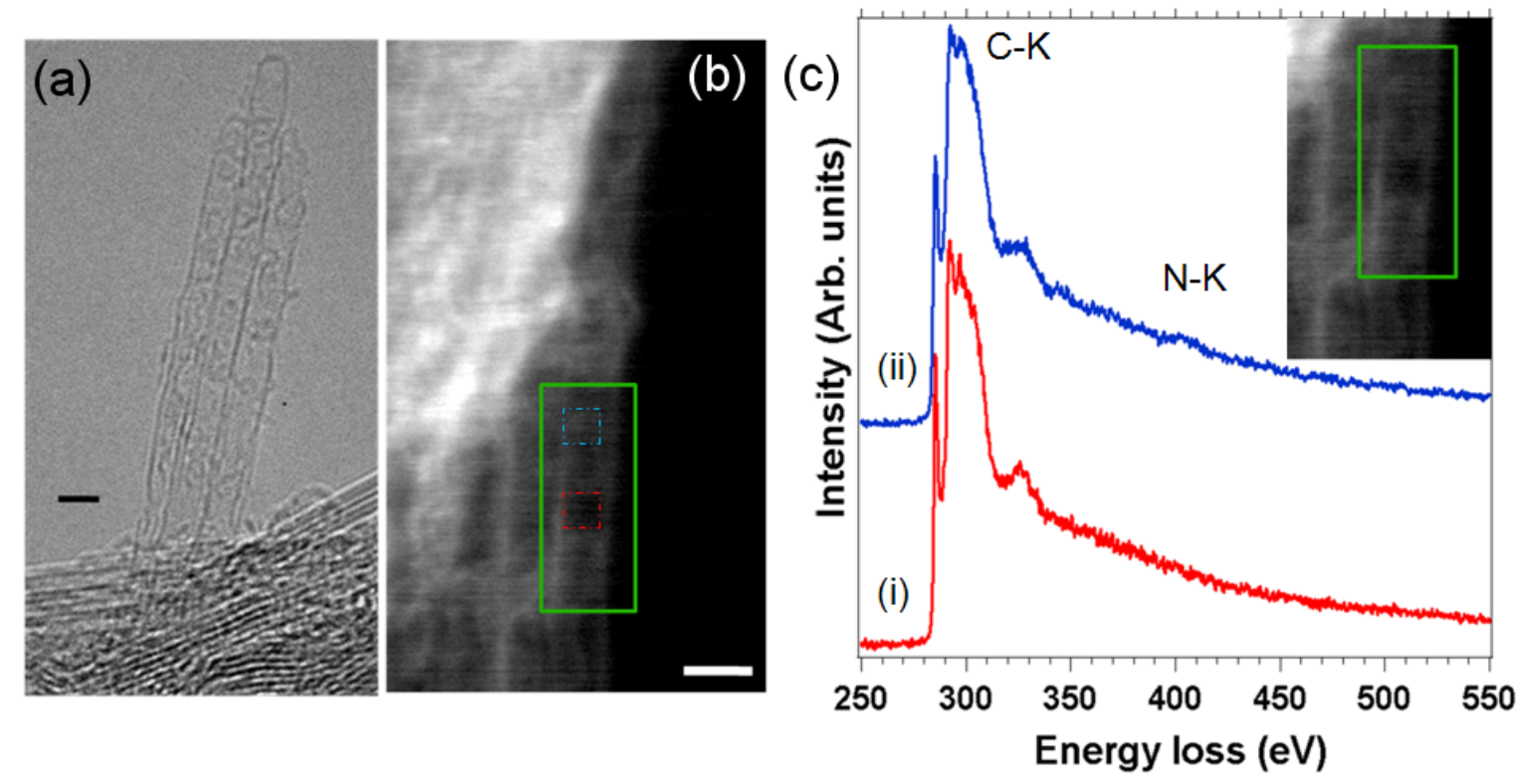

Figure 1: (a) HRTEM micrographs of bundles filled single-walled nanotubes. (b) HAADF image of another bundle of filled single-walled nanotubes. A 7x16 EELS SPIM has been recorded in the marked green area of this micrograph. (c) Sums of 9 selected EEL spectra collected from the two different marked areas in the SPIM, marked in Fig. 1.b. While the C-K edge is visible in both spectra, the N-K edge is only visible in the blue (Figure 1 (cii))EEL spectrum acquired in the blue area of the HAADF image (Figure 1.b). The insert of this figure is a zoom of the HAADF image displayed in (b). The scale bars of the two micrographs (Figure 1.a and b) are $2 \mathrm{~nm}$.

This C-K edge shows the characteristic peaks of $\mathrm{sp}^{2}$ materials at $\sim 285 \mathrm{eV}$ and $\sim 292 \mathrm{eV}$, corresponding to the $\pi^{*}$ and $\sigma^{*}$ contributions, respectively. ${ }^{30-32}$ The most significant point 
concerning these EELS results is that in the spectrum acquired over the blue region (Figure 1b), we additionally detect nitrogen (see Figure1.c-ii). This nitrogen is clearly associated to FePc, as it is the only source of nitrogen in these samples. Thus, from the combination of the HRTEM images and local analytical findings, we conclude that these observed moieties correspond to the FePc, which are localized in the hollow core of NTs. However, these investigations do not allow to determine the long-range organization of the molecules confined into nanotubes.

X-ray diffraction: The x-ray diffraction experiments are first used to investigate the structural organization of the confined species into the hollow core of the nanotubes but also to probe the encapsulation efficiency of the phthalocyanine molecules. Indeed the main peak (referred as 10 peak) arising from the two-dimensional structural organization of the nanotubes into bundles is well known to vanish under encapsulation. ${ }^{35,36}$ Independently of the central atom, phthalocyanine molecules can organize into two main structures, respectively called the $\alpha$ and the $\beta$ phases. ${ }^{2,37-46}$ The $\alpha$ structure is generally reported as metastable whereas the $\beta$ one is thermally stable over a large temperature range. ${ }^{37-40}$ Both phases are stacked molecules forming columns with the ring tilted with respect to the vertical axis. . $^{2,41,45,47}$

Figure 2 exhibits the diffraction pattern of raw and functionalized NT14 and NT17 nanotubes, the $\alpha$ and $\beta$ FePc phases. The 10 peak of the nanotube bundles is located at $5.85 \pm 0,03^{\circ}$ for raw and purified NT14 and at $4.57 \pm 0,03^{\circ}$ for purified NT17. These positions correspond respectively to inter reticular distances of $15.1 \pm 0.1$ and $19.3 \pm 0.15 \AA$, in good agreement with the tube diameter distributions. After outside functionalization $\left(\mathrm{FePc} / \mathrm{NT}_{\mathrm{ar}}\right)$, the peaks are shifted towards lower angle, respectively $5.7 \pm 0,03^{\circ}$ for NT14 and $4.2 \pm 0,03^{\circ}$ for NT17 (not shown). This modification could be explained by a very weak dilatation of the bundles. Another explanation is to assign the shift to form factor interferences as already described for iodine intercalation into nanotubes. ${ }^{35}$ Anyway, the presence of the 10 peak clearly indicates that, as expected the nanotubes are still mainly empty after outside functionalization.

After FePc encapsulation, the 10 peaks are no longer observable for FePc@NT14 and FePc@NT17, confirming the nanotube filling, which is also evidenced by SR-EELS results. Furthermore, a new broad peak is observed below $7^{\circ}$ for both samples. It turns out that both the $\alpha$ and $\beta$ FePc phases exhibit a diffraction peak located respectively around $6.9^{\circ}$ and $7^{\circ}$ (figure 2 ), and corresponding to inter-reticular distances between the stacking axes, normal to the phthalocyanine rings. ${ }^{39}$ The presence of only one peak around $6.9^{\circ}$ is generally associated to the formation of an $\alpha$-phase for $\mathrm{FePc},{ }^{42,44} \mathrm{ZnPc}^{40,45,46,48}$ and $\mathrm{H}_{2} \mathrm{Pc}^{47}$ films or crystals and corresponds to an intermolecular distance along the [200] direction. Thus, the new peak features the structural organization of the FePc molecules inside the carbon nanotubes. 


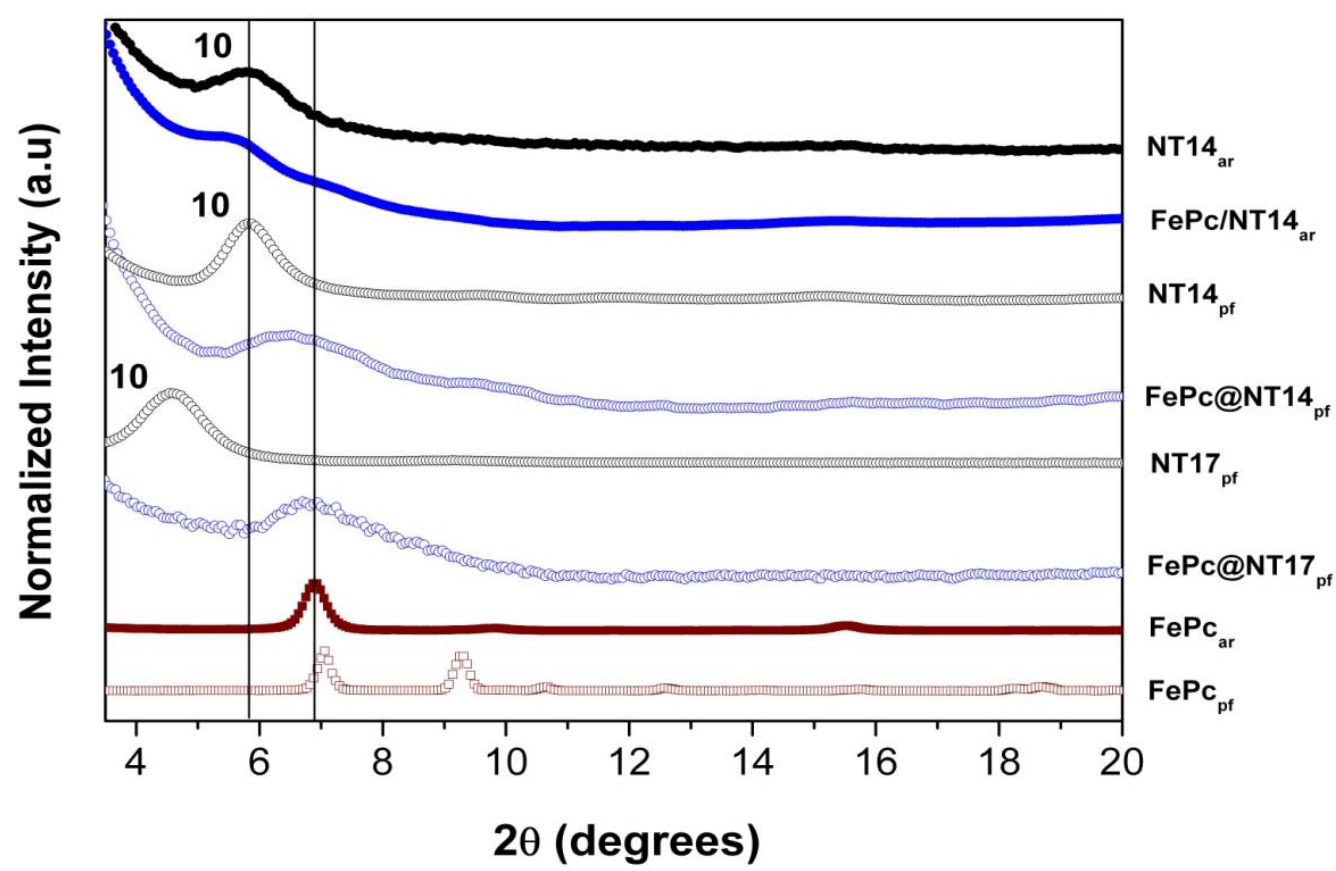

Figure 2: X-ray diffraction pattern recorded with a X-ray wavelength of $1.542 \AA$. (from top to bottom) as prepared NT14 (black full circle), FePc/NTar (full blue circle), purified NT14 (open black circle), FePc@NT14

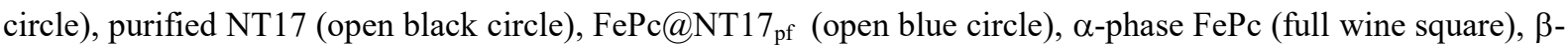
phase FePc (open wine square). The intensities are adjusted for clarity.

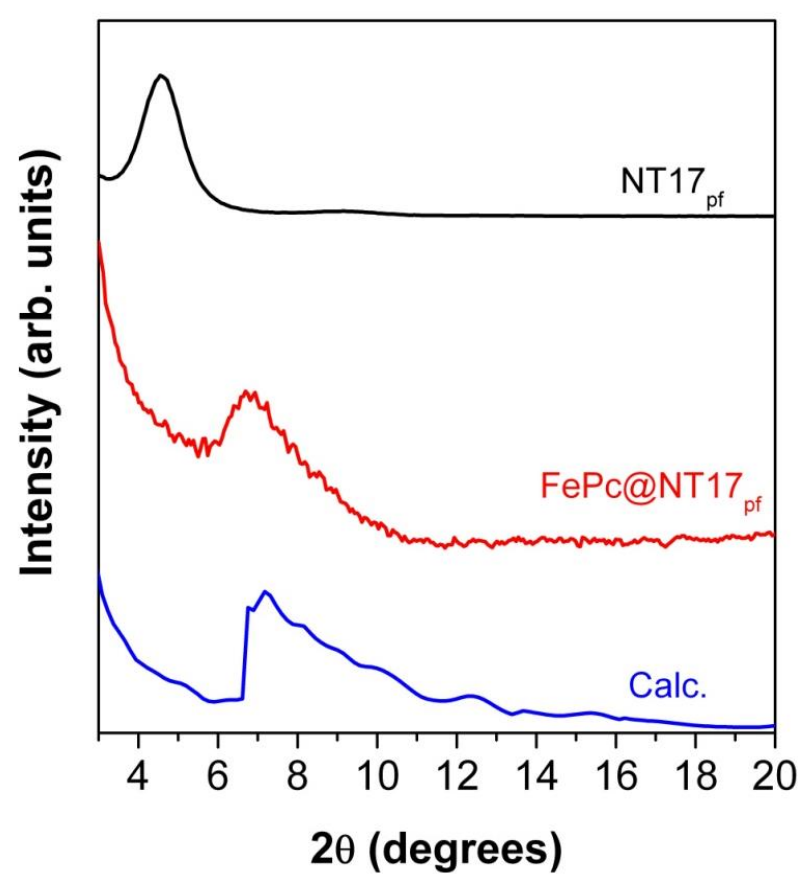

Figure 3: Experimental x-ray diffraction pattern of the reference NT17 pf powder (top) and the FePc@NT17 pf powder (center). Calculated x-ray diffraction pattern of a powder of filled NTs of diameter $1.7 \mathrm{~nm}$, with a diameter distribution of FWHM $0.17 \mathrm{~nm}$, (bottom; see experimental section for details).

To confirm our assumption on the structure of Fe-phtalocyanines inside the nanotubes, a numerical calculation of the diffraction pattern of the FePc@NT17 sample is shown on figure 3 and compared to the experimental profile. The formalism is the same as the one used in our 
previous calculations of $\mathrm{C}_{60}$ peapods ${ }^{49}$ and iodine confined in NTs. ${ }^{50}$ The intensity calculated within this model gives a very nice reproduction of the experimental diffraction profile. The 10 peak has disappeared because of the interference effects already discussed above. In the same time, an asymmetric peak of saw-tooth shape has grown, testifying for the 1D nature of the FePc stacking in NTs. Consequently, the new peak around $6.85^{\circ}$ is definitively assigned to the FePc ordering inside the nanotubes. This supramolecular organization under nano-confinement is suggested to be a 1D array of molecules lying into the nanotubes. According to the Scherrer's equation, the mean crystallite size is about $5 \mathrm{~nm}$, meaning an average cluster of around 4 molecules. It is worth mentioning that those results also stands for $\mathrm{ZnPc}$ and metal free molecules encapsulated into nanotubes.

To go further, we performed DFT-calculations to determine the molecule orientation with respect to the nanotube axis. With an intermolecular distance set at $12.8 \AA$, derived from reference ${ }^{46}$ and in good agreement with our experimental data, the minimum of the bonding energy is obtained for an angle between the molecular ring and the nanotubes axis calculated at $32^{\circ}$ as shown on figure 4. Thus, the corresponding stacking angle as defined in reference is $58^{\circ}$, quite different from the $36^{\circ}$ proposed for multi-walled nanotubes. ${ }^{20}$

Thus, nano-confinement inside single-walled nanotubes gives rise to a $1 \mathrm{D}$ supramolecular organization of the MPc molecules monitored by the small NT diameter.

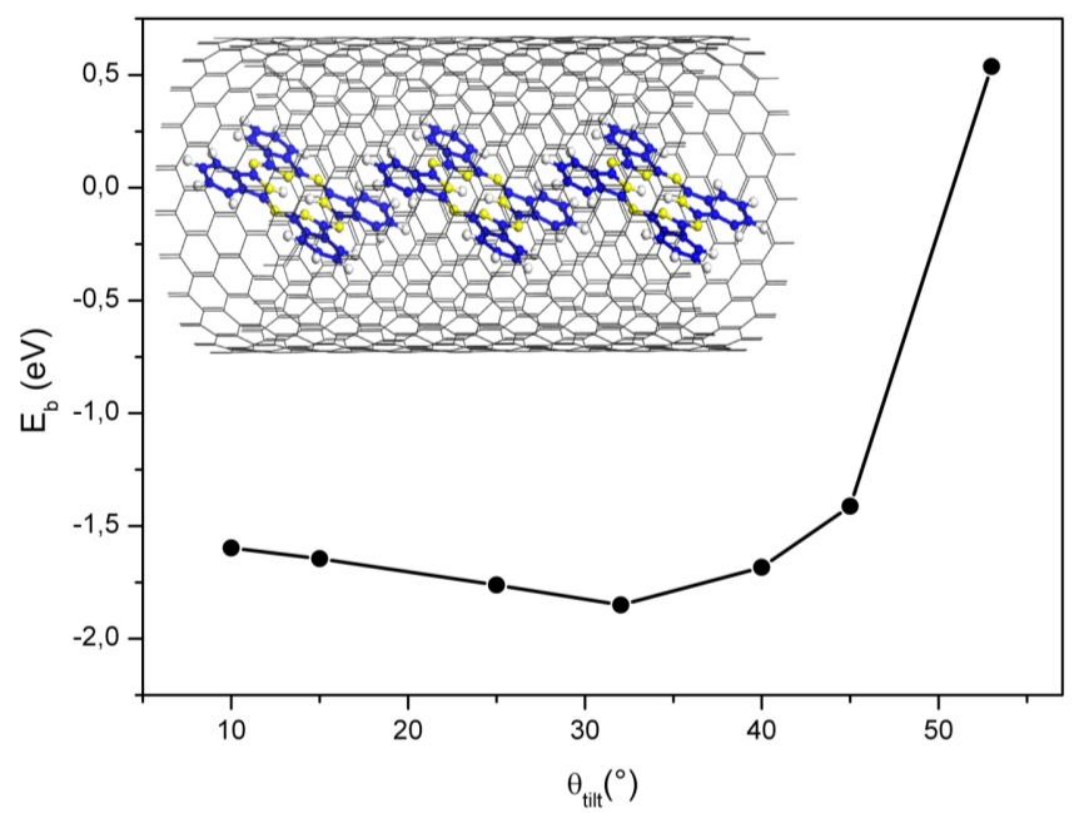

Figure 4: Binding energy as a function of the tilt angle of the Pc molecule with respect to the nanotube $(22,0)$ axis Inset: Scheme of the Pc structural organization inside single-walled carbon nanotubes $(22,0)$

\section{Raman experiments:}

The Raman spectra of the hybrid systems exhibit an important dependence with the excitation energy which is correlated to the optical absorption energy of the photo-active molecule. ${ }^{51}$ Figure 5 exhibits the Raman spectra recorded with an excitation wavelength of $633 \mathrm{~nm}$ of (a) the different purified phthalocyanine molecules MPc, (b) endohedral NT14 (MPc@NT14), and 
(c) NT14 functionalized at their outer surface (MPc/NT14). The intensities are normalized either on the G-band peak or on the most intense peak of the MPc Raman signal.

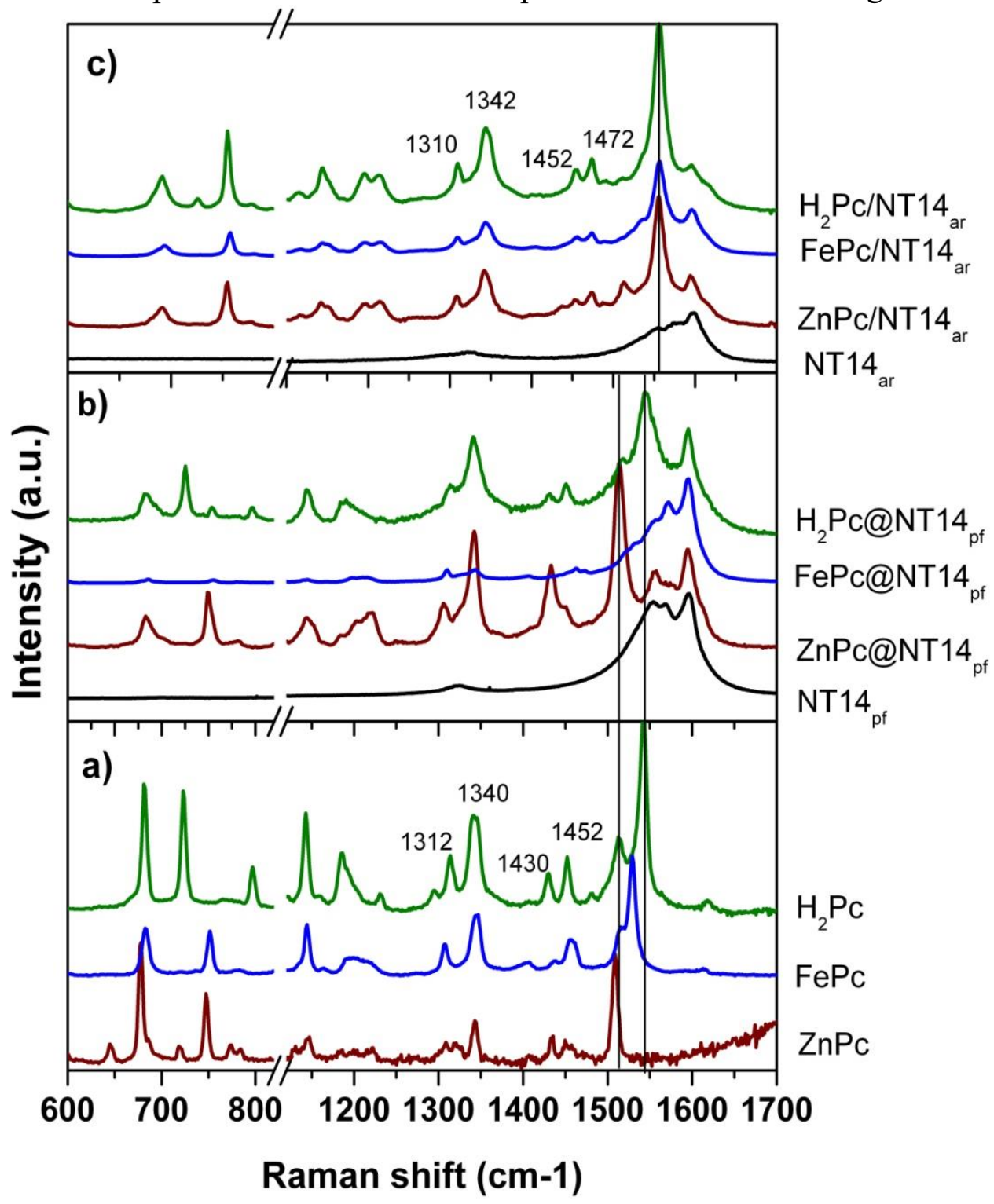

Figure 5: Normalized Raman spectra recorded with an excitation wavelength of $633 \mathrm{~nm}$ a) purified phthalocyanine molecules MPc -a strong background had to be subtracted- (b) purified NT14 $4_{\mathrm{pf}}$ and endohedral functionalized NT14 $\left(\right.$ pPc $\left.@ N T 14_{\mathrm{pf}}\right) \mathrm{c}$ ) as received NT14 $4_{\mathrm{ar}}$ and surface functionalized NT14 $4_{\mathrm{ar}}\left(\mathrm{MPc} / \mathrm{NT} 14_{\mathrm{ar}}\right)$

Figure 5.a clearly shows that the central atoms display clear fingerprints in the Raman spectra as both profiles and peak positions change depending on the type of the MPc molecules (respectively between 600 and $800 \mathrm{~cm}^{-1}$ and between 1400 and $1600 \mathrm{~cm}^{-1}$ ). The peaks around 680 and $750 \mathrm{~cm}^{-1}$ mainly correspond to breathing and deformation of the macrocycle whereas those lying between 1500 and $1550 \mathrm{~cm}^{-1}$ involve mainly C-N-C stretching. ${ }^{52-54}$ All of them concern vibrations around the metal central atom, accounting for the difference in the Raman spectra for the three different phthalocyanine molecules. Most of the peaks between 1000 and $1500 \mathrm{~cm}^{-1}$ involves mainly in-plane deformation and they are much less affected by the central atom size. ${ }^{52-56}$ 
Figure 5.b displays the Raman spectra recorded of NT14 pe nanotubes and after encapsulation (MPc@NT14pf). The nanotube G-band exhibits a Breit-Wigner-Fano profile, characteristic of metallic nanotubes and in good agreement with the Kataura plot. The photoluminescence background of MPc is no longer observable after encapsulation into nanotubes and a Raman signal is observed, demonstrating the bleaching of the MPc photoluminescence effect. Nevertheless, the Raman spectrum of the hybrid system consists roughly in the superposition the Raman spectra of the nanotube and the confined molecule. These data strongly suggests that the encapsulated MPc behaves as a "free" molecule inside the nanotubes.

Figure 5.c displays the Raman spectra of the as-received NT14 nanotubes and after noncovalent functionalization at their outer surface with phthalocyanine molecules with different central atoms (MPc/NT14). The spectra no longer consist in the superposition of the MPc molecule and the nanotube Raman responses, indicating a significant interaction between both systems. In all cases, the profiles of the Raman spectra seem very similar, independently of the central atom, meaning that its Raman fingerprint cannot be observed anymore. These results strongly suggest that the central atom does not play any important role in the molecule vibrations. However, those Raman spectra do not match the Raman spectrum of the bulk $\mathrm{H}_{2} \mathrm{Pc}$ molecules (figure 5) meaning that another effect has to be taken into account. For instance, the most intense peak is localized at $1556 \mathrm{~cm}^{-1}$ for all MPc@NTs but shifted $\left(14 \mathrm{~cm}^{-1}\right)$ compared to $\mathrm{H}_{2} \mathrm{Pc}$. Furthermore, important discrepancies are observed in the low frequency domain (below 800 $\mathrm{cm}^{-1}$ ) where out-of-plane macrocycle vibrations are probed. Our hypothesis is to consider that the MPc molecules $\pi$-stacked at the outer surface fit the nanotube curvature, so that their structures are no longer flat. As a consequence, the central atom is out-of-plane and no longer contributes to the molecule vibrations. This assumption is strongly supported by the important shift of the peaks at 1430 and $1452 \mathrm{~cm}^{-1}$ (respectively 1452 and $1472 \mathrm{~cm}^{-1}$ ) for the bulk $\mathrm{H}_{2} \mathrm{Pc}$ (respectively $\mathrm{H}_{2} \mathrm{Pc}$ stacked onto NT). Indeed, those peaks arise from the vibrations of carbon atoms within the isoindole group ${ }^{57}$ (localized near the molecule edge) and are thus strongly affected by the phthalocyanine curvature. Consequently, the differences in the Raman spectra of $\mathrm{H}_{2} \mathrm{Pc}$ and $\mathrm{H}_{2} \mathrm{Pc} / \mathrm{NT} 14$ are assigned to the $\mathrm{H}_{2} \mathrm{Pc}$ ring curvature due to the interaction with the nanotubes walls.

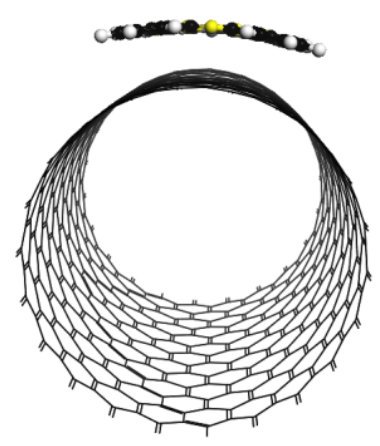

Figure 6: Structure of the ZnPc stacked at the outer surface of NT $(17,0)$ from our DFT calculations.

This assumption is consistent with DFT calculations (figure 6), showing that the ring molecules switch from an angle of $180^{\circ}$ (flat structure) to an angle of $154^{\circ}$ (curved structure on the 
nanotubes) for a $(17,0)$ nanotube. In addition, taking van der Waals interactions into account, the central atom ( $\mathrm{Zn}$ in our model) moves slightly towards the nanotube wall, being thus out of the molecule plane (figure 7). Let's consider the curved ZnPc stacked onto the nanotubes (upleft corner of figure 7) and only some of its atoms (those inside a black open circle on the upright corner). Figure 7 displays the projection of those peculiar atoms onto the (xy) plane. The $\mathrm{Zn}$ atom is slightly but clearly shifted upon interaction with the tube. Thus, we assume that the central atom no longer plays a role in the molecule vibration, accounting for our Raman data.

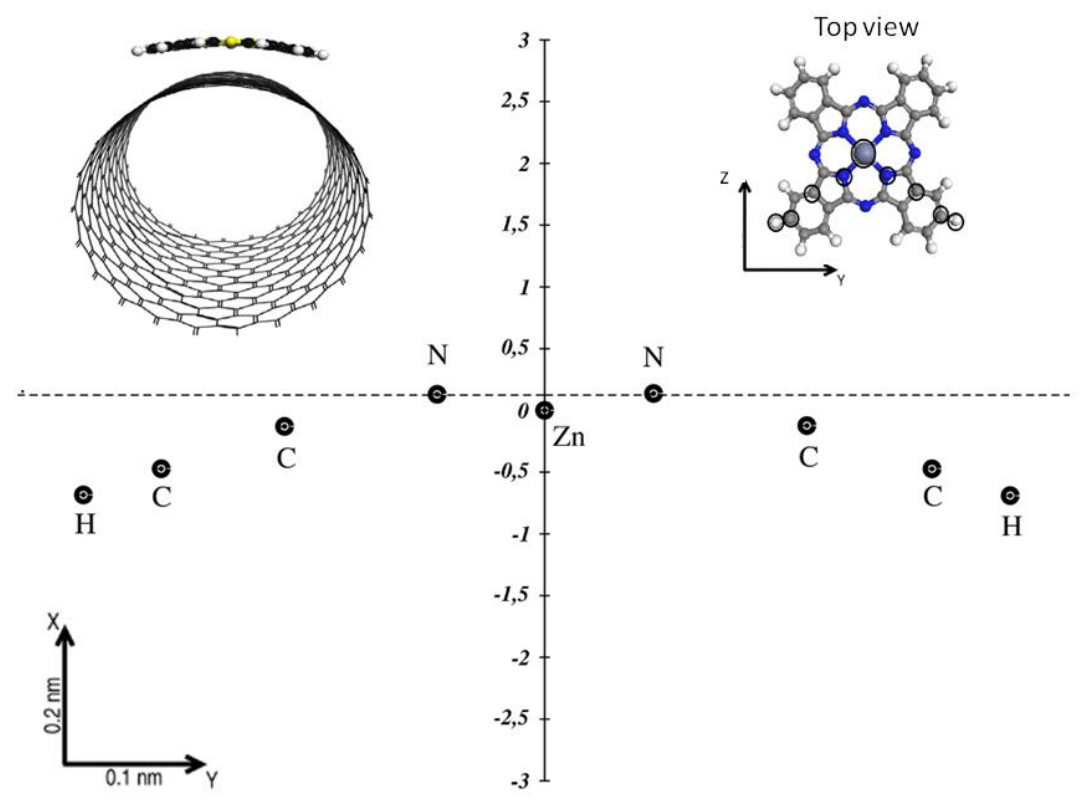

Figure 7: Projection onto the $(\mathrm{x}, \mathrm{y})$ plane of atoms of the $\mathrm{ZnPc}$ molecule stacked onto a nanotube .

To go further, we also studied hybrid systems with carbon structures displaying different curvatures (graphite, as received NT17 and NT14 nanotubes). Figure 8 exhibits the Raman spectra of bulk FePc and molecules deposited on these different carbon structures. As previously observed, stacking on NT14 ar gives rise to important changes in the FePc Raman response (peak shifted from 1510 to $1540 \mathrm{~cm}^{-1}$ and splitting of the peak around $1450 \mathrm{~cm}^{-1}$ ). By contrast, the FePc vibrations do not look altered by the interaction with graphite or NT17 ar. One can assume that the curvatures of the host carbon materials are not large enough to induce significant modifications of the FePc structure (remaining flat), preventing any change in the Raman vibrations.

One can notice that according to the Kataura plot, NT14 and NT17 nanotubes are respectively metallic and semiconducting for a $660 \mathrm{~nm}$ excitation wavelength (whereas graphite is a semimetal). As similar results are obtained for both semiconducting and metallic NT17 with excitation wavelengths of respectively 660 and $785 \mathrm{~nm}$, we assume that the electronic properties do not play significant role in the interaction and that the nanotube diameter is the relevant parameter. 


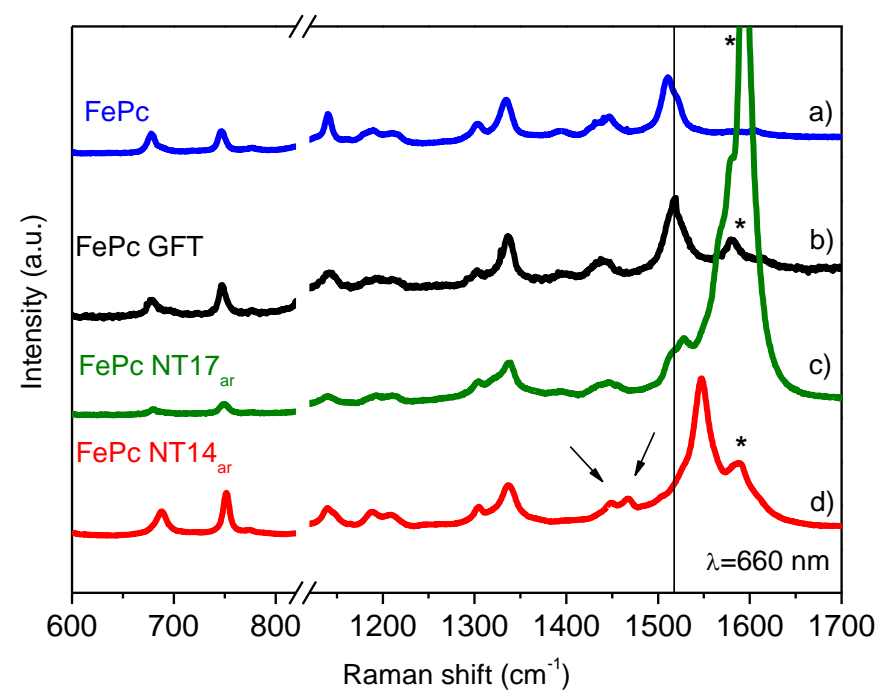

Figure 8: Raman spectra recorded with an excitation wavelength of $660 \mathrm{~nm}$ a) purified phthalocyanine molecules MPc (b) FePc/Graphite c) FePc/NT17 ar d) FePc/NT14 ar * Indicates the the G-band of the host carbon materials

\section{Conclusions:}

Phthalocyanine molecules have been noncovalently functionalized inside or at the outer surface of single-walled carbon nanotubes. HRTEM and SR-EELS analyses have confirmed that the MPc molecules are located inside of nanotubes. The structural 1D organization of the molecules inside the nanotubes is determined by $\mathrm{x}$-ray diffraction coupled to DFT calculations. The MPc molecules are stacked along the tube axis, with an angle of $32^{\circ}$, giving rise to a 1D phase under nano-confinement. The Raman data suggest only a very weak interaction when the molecules are confined into the nanotubes. By contrast, localized at the outer surface, a strong interaction takes place. The strength of this last interaction depends on the nanotube diameter and induces a significant curvature of the molecule ring when decreasing the NT diameter. As a consequence, the central atom (M) gets out of plane, impacting the molecule vibration properties. These results demonstrate that carbon nanotubes can play the role of nano-support for organic molecules of interest in opto-electronic applications. As a matter of facts, they can guide their linear organization and can even bend them to an extent defined by the nanotubes radius. These results open exciting perspectives for the development of new hybrid systems with tunable optical properties.

\section{Acknowledgments:}

The transmission electron microscopy studies were conducted at the Laboratorio de MicroscopiasAvanzadas (LMA) at the Instituto de Nanociencia de Aragon (INA) - Universidad de Zaragoza (Spain). The TEM measurements were supported by the European Union Seventh 
Framework Program under Grant Agreement 312483 - ESTEEM2 (Integrated Infrastructure Initiative - I3).

\section{References:}

(1) Heutz, S.; Mitra, C.; Wu, W.; Fisher, A. J.; Kerridge, A.; Stoneham, M.; Harker, A. H.; Gardener, J.; Tseng, H. H.; Jones, T. S.; Renner, C.; Aeppli, G. Molecular Thin Films: A New Type of Magnetic Switch. Adv. Mater. 2007, 19, 3618-3622.

(2) Claessens, C. G.; Hahn, U.; Torres, T. Phthalocyanines: From Outstanding Electronic Properties to Emerging Applications. Chem. Rec. 2008, 8, 75-97.

(3) Imahori, H.; Umeyama, T.; Ito, S. Large $\pi$-Aromatic Molecules as Potential Sensitizers for Highly Efficient Dye-Sensitized Solar Cells. Acc. Chem. Res. 2009, 42, 1809-1818.

(4) Hains, A. W.; Liang, Z.; Woodhouse, M. A.; Gregg, B. A. Molecular Semiconductors in Organic Photovoltaic Cells. Chem. Rev. 2010, 110, 6689-6735.

(5) Bottari, G.; de la Torre, G.; Guldi, D. M.; Torres, T. Covalent and Noncovalent Phthalocyanine-Carbon Nanostructure Systems: Synthesis, Photoinduced Electron Transfer, and Application to Molecular Photovoltaics. Chem. Rev. 2010, 110, 6768-6816.

(6) Kyatskaya, S.; Mascarós, J. R. G.; Bogani, L.; Hennrich, F.; Kappes, M.; Wernsdorfer, W.; Ruben, M. Anchoring of Rare-Earth-Based Single-Molecule Magnets on Single-Walled Carbon Nanotubes. J. Am. Chem. Soc. 2009, 131, 15143-15151.

(7) Gimenez-Lopez, M. d. C.; Moro, F.; La Torre, A.; Gomez-Garcia, C. J.; Brown, P. D.; van Slageren, J.; Khlobystov, A. N. Encapsulation of Single-molecule Magnets in Carbon Nanotubes. Nat. Commun. 2011, 2, 407.

(8) Mativetsky, J. M.; Wang, H.; Lee, S. S.; Whittaker-Brooks, L.; Loo, Y.-L. Face-on Stacking and Enhanced Out-of-plane Hole Mobility in Graphene-templated Copper Phthalocyanine. Chem. Commun. 2014, 50, 5319-5321.

(9) Campidelli, S.; Ballesteros, B.; Filoramo, A.; Díaz, D. D.; de la Torre, G.; Torres, T.; Rahman, G. M. A.; Ehli, C.; Kiessling, D.; Werner, F. et al. Facile Decoration of Functionalized Single-Wall Carbon Nanotubes with Phthalocyanines via "Click Chemistry". J. Am. Chem. Soc. 2008, 130, 11503-11509.

(10) Wang, Y.; Hu, N.; Zhou, Z.; Xu, D.; Wang, Z.; Yang, Z.; Wei, H.; Kong, E. S.-W.; Zhang, Y. Singlewalled Carbon Nanotube/Cobalt Phthalocyanine Derivative Hybrid Material: Preparation, Characterization and Its Gas Sensing Properties. J. Mater. Chem. 2011, 21, 3779-3787.

(11) Ganzhorn, M.; Klyatskaya, S.; Ruben, M.; Wernsdorfer, W. Carbon Nanotube Nanoelectromechanical Systems as Magnetometers for Single-Molecule Magnets. ACS Nano 2013, 7, 6225-6236.

(12) Bazarnik, M.; Brede, J.; Decker, R.; Wiesendanger, R. Tailoring Molecular Self-Assembly of Magnetic Phthalocyanine Molecules on Fe- and Co-Intercalated Graphene. ACS Nano 2013, 7, 1134111349.

(13) Ragoussi, M.-E.; Katsukis, G.; Roth, A.; Malig, J.; de la Torre, G.; Guldi, D. M.; Torres, T. Electron-Donating Behavior of Few-Layer Graphene in Covalent Ensembles with Electron-Accepting Phthalocyanines. J. Am. Chem. Soc. 2014, 136, 4593-4598.

(14) Ince, M.; Bartelmess, J.; Kiessling, D.; Dirian, K.; Martinez-Diaz, M. V.; Torres, T.; Guldi, D. M. Immobilizing NIR Absorbing Azulenocyanines onto Single Wall Carbon Nanotubes-from Charge Transfer to Photovoltaics. Chem. Sci. 2012, 3, 1472-1480.

(15) Bartelmess, J.; Ballesteros, B.; de la Torre, G.; Kiessling, D.; Campidelli, S.; Prato, M.; Torres, T.; Guldi, D. M. Phthalocyanine-Pyrene Conjugates: A Powerful Approach toward Carbon Nanotube Solar Cells. J. Am. Chem. Soc. 2010, 132, 16202-16211.

(16) Morozan, A.; Campidelli, S.; Filoramo, A.; Jousselme, B.; Palacin, S. Catalytic Activity of Cobalt and Iron Phthalocyanines or Porphyrins Supported on Different Carbon Nanotubes Towards Oxygen Reduction Reaction. Carbon 2011, 49, 4839-4847. 
(17) Cao, R.; Thapa, R.; Kim, H.; Xu, X.; Kim, M. G.; Li, Q.; Park, N.; Liu, M.; Cho, J. Promotion of Oxygen Reduction by a Bio-inspired Tethered Iron Phthalocyanine Carbon Nanotube-based Catalyst. Nat. Commun. 2013, 4, 2076.

(18) Urdampilleta, M.; Klyatskaya, S.; Cleuziou, J. P.; Ruben, M.; Wernsdorfer, W. Supramolecular Spin Valves. Nat. Mater. 2011, 10, 502-506.

(19) Lopes, M.; Candini, A.; Urdampilleta, M.; Reserbat-Plantey, A.; Bellini, V.; Klyatskaya, S.; Marty, L.; Ruben, M.; Affronte, M.; Wernsdorfer et al. Surface-Enhanced Raman Signal for Terbium Single-Molecule Magnets Grafted on Graphene. ACS Nano 2010, 4, 7531-7537.

(20) Swarbrick, J. C.; Weng, T.-C.; Schulte, K.; Khlobystov, A. N.; Glatzel, P. Electronic Structure Changes in Cobalt Phthalocyanine Due to Nanotube Encapsulation Probed Using Resonant Inelastic X-ray Scattering. Phys. Chem. Chem. Phys. 2010, 12, 9693-9699.

(21) Schulte, K.; Swarbrick, J. C.; Smith, N. A.; Bondino, F.; Magnano, E.; Khlobystov, A. N. Assembly of Cobalt Phthalocyanine Stacks inside Carbon Nanotubes. Adv. Mater. 2007, 19, 33123316.

(22) Saito, T.; Ohshima, S.; Okazaki, T.; Ohmori, S.; Yumura, M.; lijima, S. Selective Diameter Control of Single-Walled Carbon Nanotubes in the Gas-Phase Synthesis. J. Nanosci. Nanotechnol. 2008, 8, 6153-6157.

(23) Jeanguillaume, C.; Colliex, C. Spectrum-image: The next Step in EELS Digital Acquisition and Processing. Ultramicroscopy 1989, 28, 252-257.

(24) Arenal, R.; de la Peña, F.; Stéphan, O.; Walls, M.; Tencé, M.; Loiseau, A.; Colliex, C. Extending the Analysis of EELS Spectrum-imaging Data, from Elemental to Bond Mapping in Complex Nanostructures Ultramicroscopy 2008, 109, 32-38.

(25) Sanchez-Portal, D.; Ordejon, P.; Artacho, E.; Soler, J. M. Density-functional Method for Very Large Systems with LCAO Basis Sets. Int. J. Quantum Chem. 1997, 65, 453-461.

(26) Perdew, J. P.; Burke, K.; Ernzerhof, M. Generalized Gradient Approximation Made Simple. Phys. Rev. Lett. 1996, 77, 3865-3868.

(27) Troullier, N.; Martins, J. L. Efficient Pseudopotentials for Plane-wave Calculations. Phys. Rev. B 1991, 43, 1993-2006.

(28) Artacho, E.; Sanchez-Portal, D.; Ordejon, P.; Garcia, A.; Soler, J. M. Linear-scaling Ab-initio Calculations for Large and Complex Systems. Phys. Status Solidi B 1999, 215, 809-817.

(29) Grimme, S. Semi-empirical GGA-type Density Functional Constructed with a Long-range Dispersion Correction. J. Comput. Chem. 2006, 27, 1787-1799.

(30) Ayala, P.; Arenal, R.; Ruemmeli, M.; Rubio, A.; Pichler, T. The Doping of Carbon Nanotubes with Nitrogen and their Potential Applications. Carbon 2010, 48, 575-586.

(31) Ayala, P.; Arenal, R.; Loiseau, A.; Rubio, A.; Pichler, T. The Physical and Chemical Properties of Heteronanotubes. Rev. Mod. Phys. 2010, 82, 1843-1885.

(32) Arenal, R.; Blase, X.; Loiseau, A. Boron-nitride and Boron-carbonitride Nanotubes: Synthesis, Characterization and Theory. Adv. Phys. 2010, 59, 101-179.

(33) Arenal, R.; De Matteis, L.; Custardoy, L.; Mayoral, A.; Tence, M.; Grazu, V.; De La Fuente, J. M.; Marquina, C.; Ibarra, M. R. Spatially-Resolved EELS Analysis of Antibody Distribution on Biofunctionalized Magnetic Nanoparticles. ACS Nano 2013, 7, 4006-4013.

(34) Alvarez, L.; Almadori, Y.; Arenal, R.; Babaa, R.; Michel, T.; Le Parc, R.; Bantignies, J. L.; Jousselme, B.; Palacin, S.; Hermet, $\mathrm{P}$ et al. Charge Transfer Evidence between Carbon Nanotubes and Encapsulated Conjugated Oligomers. J. Phys. Chem. C 2011, 115, 11898-11905.

(35) Bendiab, N.; Almairac, R.; Rols, S.; Aznar, R.; Sauvajol, J. L.; Mirebeau, I. Structural Determination of lodine Localization in Single-walled Carbon Nanotube Bundles by Diffraction Methods. Phys. Rev. B 2004, 69, 195415.

(36) Maniwa, Y.; Kumazawa, Y.; Saito, Y.; Tou, H.; Kataura, H.; Ishii, H.; Suzuki, S.; Achiba, Y.; Fujiwara, A.; Suematsu, H. Anomaly of X-ray Diffraction Profile in Single-walled Carbon Nanotubes. Jpn. J. Appl. Phys. 1999, 38, L668-L670.

(37) El-Nahass, M. M.; Bahabri, F. S.; Ghamdi, A. A. A.; Al-Harbi, S. R. Structural and Transport Properties of Copper Phthalocyanine (CuPc) Thin Films. Egypt. J. Sol. 2002, 25, 307-321. 
(38) Ballirano, P.; Caminiti, R.; Ercolani, C.; Maras, A.; Orrù, M. A. X-ray Powder Diffraction Structure Reinvestigation of the $\alpha$ and $\beta$ Forms of Cobalt Phthalocyanine and Kinetics of the $\alpha \rightarrow \beta$ Phase Transition. J. Am. Chem. Soc. 1998, 120, 12798-12807.

(39) Senthilarasu, S.; Baek, S.-J.; Chavhan, S. D.; Lee, J.; Lee, S.-H. Effect of Temperature on Stacking Orientations of Zinc Phthalocyanine Thin Films. J. Nanosci. Nanotechnol. 2008, 8, 5414-5417.

(40) Senthilarasu, S.; Hahn, Y. B.; Lee, S.-H. Structural Analysis of Zinc Phthalocyanine (ZnPc) Thin Films: X-ray Diffraction Study. J. Appl. Phys. 2007, 102, 043512.

(41) Miller, C. W.; Sharoni, A.; Liu, G.; Colesniuc, C. N.; Fruhberger, B.; Schuller, I. K. Quantitative Structural Analysis of Organic Thin Films: An X-ray Diffraction Study. Phys. Rev. B 2005, 72, 104113.

(42) Baranton, S.; Coutanceau, C.; Garnier, E.; Leger, J. M. How Does Alpha-FePc Catalysts Dispersed onto High Specific Surface Carbon Support Work Towards Oxygen Reduction Reaction (ORR)? J. Electroanal. Chem. 2006, 590, 100-110.

(43) Kment, S.; Kluson, P.; Drobek, M.; Kuzel, R.; Gregora, I.; Kohout, M.; Hubicka, Z. Preparation of Thin Phthalocyanine Layers and their Structural and Absorption Properties. Thin Solid Films 2009, 517, 5274-5279.

(44) Debnath, A. K.; Samanta, S.; Singh, A.; Aswal, D. K.; Gupta, S. K.; Yakhmi, J. V.; Deshpande, S. K.; Poswal, A. K.; Surgers, C. Growth of Iron Phthalocyanine Nanoweb and Nanobrush Using Molecular Beam Epitaxy. Physica E 2008, 41, 154-163.

(45) Zanfolim, A. A.; Volpati, D.; Olivati, C. A.; Job, A. E.; Constantino, C. J. L. Structural and Electric-Optical Properties of Zinc Phthalocyanine Evaporated Thin Films: Temperature and Thickness Effects. J. Phys. Chem. C 2010, 114, 12290-12299.

(46) Yamane, H.; Hatsui, T.; Iketaki, K.; Kaji, T.; Hiramoto, M.; Kosugi, N. Site-specific Intermolecular Interaction in Alpha-phase Crystalline Films of Phthalocyanines Studied by Soft X-ray Emission Spectroscopy. J. Chem. Phys. 2011, 135, 034704.

(47) M. Bayliss, S.; Heutz, S.; Rumbles, G.; S. Jones, T. Thin Film Properties and Surface Morphology of Metal Free Phthalocyanine Films Grown by Organic Molecular Beam Deposition. Phys. Chem. Chem. Phys. 1999, 1, 3673-3676.

(48) Scheidt, W. R.; Dow, W. Molecular Stereochemistry of Phthalocyanatozinc(II). J. Am. Chem. Soc. 1977, 99, 1101-1104.

(49) Cambedouzou, J.; Pichot, V.; Rols, S.; Launois, P.; Petit, P.; Klement, R.; Kataura, H.; Almairac, R. On the Diffraction Pattern of $C_{60}$ Peapods. Eur. Phys. J. B 2004, 42, 31-45.

(50) Chorro, M.; Kane, G.; Alvarez, L.; Cambedouzou, J.; Paineau, E.; Rossberg, A.; Kociak, M.; Aznar, R.; Pascarelli, S.; Launois, P.et al. 1D-confinement of Polyiodides Inside Single-wall Carbon Nanotubes. Carbon 2013, 52, 100-108.

(51) Alvarez, L.; Almadori, Y.; Mariot, S.; Aznar, R.; Arenal, R.; Michel, T.; Le Parc, R.; Dieudonne, P.; Jousselme, B.; Campidelli, S. et al. New Insigths on Photo-Active Molecules Within Host Carbon Nanotubes. J. Nanoelectron. Optoelectron. 2013, 8, 28-35.

(52) Saini, G. S. S.; Sukhwinder, S.; Sarvpreet, K.; Ranjan, K.; Vasant, S.; Tripathi, S. K. Zinc Phthalocyanine Thin Film and Chemical Analyte Interaction Studies by Density Functional Theory and Vibrational Techniques. J. Phys.: Condens. Matter 2009, 21, 225006.

(53) Liu, Z.; Zhang, X.; Zhang, Y.; Jiang, J. Theoretical Investigation of the Molecular, Electronic Structures and Vibrational Spectra of a Series of First Transition Metal Phthalocyanines. Spectrochim. Acta A 2007, 67, 1232-1246.

(54) Tackley, D. R.; Dent, G.; Ewen Smith, W. IR and Raman Assignments for Zinc Phthalocyanine from DFT Calculations. Phys. Chem. Chem. Phys. 2000, 2, 3949-3955.

(55) Heutz, S.; Salvan, G.; Silaghi, S. D.; Jones, T. S.; Zahn, D. R. T. Raman Scattering as a Probe of Crystallinity in PTCDA and $\mathrm{H}_{2} \mathrm{Pc}$ Single-Layer and Double-Layer Thin Film Heterostructures. J. Phys. Chem. B 2003, 107, 3782-3788.

(56) Szybowicz, M.; Makowiecki, J. Orientation Study of Iron Phthalocyanine (FePc) Thin Films Deposited on Silicon Substrate Investigated by Atomic Force Microscopy and Micro-raman Spectroscopy J. Mater. Sci. 2012, 47, 1522-1530. 
(57) Basova, T. V.; Kiselev, V. G.; Schuster, B.-E.; Peisert, H.; Chassé, T. Experimental and Theoretical Investigation of Vibrational Spectra of Copper Phthalocyanine: Polarized Single-crystal Raman Spectra, Isotope Effect and DFT Calculations. J. Raman Spectrosc. 2009, 40, 2080-2087.

TOC

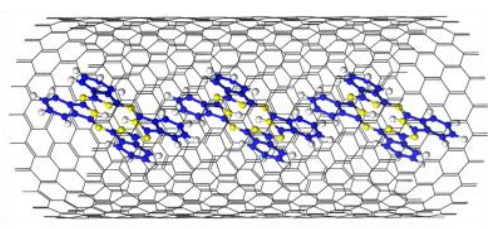


Figure 1: (a) HRTEM micrograph of bundles of filled single-walled nanotubes. (b) HAADF image of another bundle of filled single-walled nanotubes. A 7x16 EELS SPIM has been recorded in the marked green area of this micrograph. (c) Sums of 9 selected EEL spectra collected from the two different marked areas in the SPIM, marked in Fig. 1.b. While the C-K edge is visible in both spectra, the N-K edge is only visible in the blue (Fig. 1 (c-ii))EEL spectrum acquired in the blue area of the HAADF image (Fig. 1.b). The insert of this figure is a zoom of the HAADF image displayed in (b). The scale bars of the two micrographs (Fig. 1.a and b) are $2 \mathrm{~nm}$.

Figure 2: X-ray diffraction pattern recorded with a X-ray wavelength of $1.542 \AA$. (from top to bottom) as prepared NT14 (black full circle), FePc/NT ar (full blue circle), purified NT14 (open black circle), FePc@NT14 pf (open blue circle), purified NT17 (open black circle), FePc@NT17 pf (open blue circle), $\alpha$-phase FePc (full wine square), $\beta$ phase FePc (open wine square). The intensities are adjusted for clarity.

Figure 3: Experimental x-ray diffraction pattern of the reference NT17 $7_{\mathrm{pf}}$ powder (top) and the FePc@NT17 powder (center). Calculated x-ray diffraction pattern of a powder of filled NTs of diameter $1.7 \mathrm{~nm}$, with a diameter distribution of FWHM $0.17 \mathrm{~nm}$, (bottom; see experimental section for details).

Figure 4: Binding energy as a function of the tilt angle of the Pc molecule with respect to the nanotube $(22,0)$ axis Inset: Scheme of the Pc structural organization inside single-walled carbon nanotubes $(22,0)$

Figure 5: Normalized Raman spectra recorded with an excitation wavelength of $633 \mathrm{~nm}$ a) purified phthalocyanine molecules MPc -a strong background had to be subtracted- (b) purified NT14 $4_{\mathrm{pf}}$ and endohedral

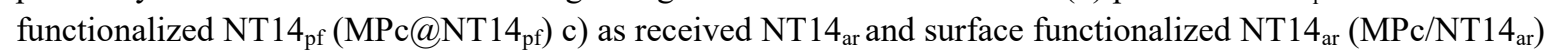

Figure 6: Structure of the ZnPc stacked at the outer surface of NT $(17,0)$ from our DFT calculations.

Figure 7: Projection onto the (x,y) plane of atoms of the $\mathrm{ZnPc}$ molecule stacked onto a nanotube .

Figure 8: Raman spectra recorded with an excitation wavelength of $660 \mathrm{~nm}$ a) purified phthalocyanine molecules MPc (b) FePc/Graphite c) FePc/NT17 ar d) FePc/NT14ar * Indicates the the G-band of the host carbon materials 